\title{
Damping of vibrations in superconducting quarter wave resonators
}

\author{
Liu Yang, ${ }^{1}$ Vladimir Zvyagintsev, ${ }^{2, *}$ R. E. Laxdal, ${ }^{2}$ and Bhalwinder Waraich ${ }^{2}$ \\ ${ }^{1}$ Institute of Fluid Physics, China Academy of Engineering Physics, \\ P.O. Box 919-106, Mianyang 621900, China \\ ${ }^{2}$ TRIUMF, 4004 Westbrook Mall, Vancouver, British Columbia, Canada V6T2A3
}

(Received 6 September 2018; published 19 March 2019)

\begin{abstract}
Ambient mechanical vibrations could cause significant detuning in superconducting accelerating cavities, disturbing the acceleration regime. The mechanical damper, first developed at Laboratori Nazionali di Legnaro, Istituto Nazionale di Fisica Nucleare by A. Facco [Mechanical mode damping in superconducting low $\beta$ resonators, in Proceedings of the Eighth Workshop on RF Superconductivity, SRF'97, Abano Terme (Padova), Italy, 1997 (JACoW, Geneva, 1997)], dissipates the kinetic energy of vibrations due to friction. The study of damping efficiency, corresponding to the maximum cavity detuning, is addressed in this paper. The mechanism of the damper parameters impacting on the maximum cavity detuning at a given excitation is investigated. An analytical model of the damper has been derived to predict the nonlinear response. Numerical results from simulations in ANSYS confirmed the model over a wide range of excitation. An experimental demonstration has been conducted successfully on a test bench. Online measurements taken on the ISAC-II superconducting linac at TRIUMF further verify the analytical model.
\end{abstract}

DOI: 10.1103/PhysRevAccelBeams.22.030103

\section{INTRODUCTION}

The coaxial quarter wave resonator (QWR) is widely used in the low $\beta$ section of heavy ion superconducting accelerators [1-5] and in addition has been developed in a deflecting mode geometry for high-luminosity colliders [6] and even as a superconducting $\mathrm{rf}$ gun [7]. Its operational $\mathrm{rf}$ mode resonated in a quarter wavelength brings compactness but also an up and down asymmetry, which poses issues of beam steering as addressed in Refs. [8,9] and operation stability [10]. Because of the inner conductor of the QWR acting like a long pendulum mostly at a frequency of 50-100 Hz, ambient microphonic noise can easily excite resonant mechanical mode vibrations. Under the natural damping of the cavity material, niobium, in general, large amplitude vibrations are likely to be developed. Correspondingly, the long decay time leads to the resonant vibrations enduring for seconds. The induced detuning from microphonic noise could bring the rf resonant frequency of the cavity outside the bandwidth of the low-level rf (LLRF) control system operation. Unlocking in the accelerating field occurs often as a result. Sufficient overcoupling of power couplers is required to

\footnotetext{
*Corresponding author. zvyagint@triumf.ca

Published by the American Physical Society under the terms of the Creative Commons Attribution 4.0 International license. Further distribution of this work must maintain attribution to the author(s) and the published article's title, journal citation, and DOI.
}

broaden the loaded bandwidth to maintain stable rf control system operation in superconducting cavities [11]. But a broader bandwidth proportionally takes more rf power to generate the necessary field level in a cavity. Efforts on complicated detuning compensation by using reactive devices or a fast tuner have been made to allow operation with less overcoupling [12-14]. On the other hand, a kind of passive friction damper was first developed at Laboratori Nazionali di Legnaro, Istituto Nazionale di Fisica Nucleare (INFN-LNL) [10]. At $4.2 \mathrm{~K}$, the options for the dissipation of vibration energy are limited in the liquid helium bath due to practical concerns of reliability. Frictional damping turns out to be a natural choice. Besides the INFN-LNL frictional damper, stainless balls inside of the inner conductors of QWRs at Inter University Accelerator Center have also been employed [15].

The INFN-LNL damper design has been employed in several accelerator projects [3-5], including the ISAC-II linac at TRIUMF, which consists of 20 superconducting QWRs operating at $106 \mathrm{MHz}$ [3] and 20 QWRs at $141 \mathrm{MHz}$. However, the damper parameters for the best damping efficiency were not optimized for the design of the ISAC-II QWRs. To generate $1 \mathrm{MV}$ accelerating voltage in a $106 \mathrm{MHz}$ QWR, $7 \mathrm{~W}$ of rf power is required for the cavity while beam loading is negligible. To broaden the $\mathrm{rf}$ bandwidth, overcoupling is used with a forward power of $\sim 200 \mathrm{~W}$ typically required. To compensate detuning, the amplitude loop controller in the LLRF system increases the rf power so that at the limits of the bandwidth a forward power of $400 \mathrm{~W}$ is required. Higher forward power, in particular, with a high standing wave ratio, increases the 
risk of overheating and rf discharge in $\mathrm{rf}$ cables of the transmission lines as well as in couplers. As a result of the discharge, there is the risk of contamination into superconducting cavities for common vacuum design cryomodules or of permanent damage to the rf feed internal to the cryomodule. Consequently, major intervention is necessary afterwards. Rather than moving towards more robust and expensive rf components, optimizing damping efficiency is favorable so that the overcoupling can be reduced.

The damping effect is represented by an equivalent mechanical quality factor to characterize the nonlinear behavior of a damped QWR structure in Ref. [10]. The derived quality factor is proportional to the vibration amplitude and has an inverse proportionality to the damper mass. However, this result is more applicable to the scenario assuming free slipping over an oscillation cycle, though a partial slip was considered in the moderate excitation regime. In the scope of free slipping, the energy dissipation by friction is proportional to the vibration amplitude, while the stored energy is proportional to the square of the amplitude. There will be a tendency of stronger vibration and less damping for the system response. The system could turn into an unbounded instability. This paper presents a study to understand the key factors impacting on damping efficiency and the parameters controlling the maximum detuning level in the operational condition. The analytical model developed in this paper to address the issue of nonlinear system response differs from the approach in Ref. [10] in three important aspects. First, a time-varying model characterizing the relative motion on the friction interface in an oscillation cycle is introduced to derive the response at a moderate excitation. This is crucial for understanding the mechanism of the damper parameters impacting on the maximum cavity detuning. Second, the excitation that defines the boundary of a damping effective region is identified as a criterion of recognizing system instability. Third, the model is further extended to solve the response involving a two-dimensional effect, which is arisen from the excitation in mutually orthogonal directions. The paper is organized in the following manner. Section II derives a theoretical model to predict the response over a wide range of excitations. Section III covers simulations in ANSYS performed to confirm the numerical result and to capture the transient response in the time domain. Experiments conducted on a bench setup to demonstrate the theoretical model are reported in Sec. IV. Online measurements on the ISAC-II linac are presented in Sec. V, and conclusions are given in Sec. VI.

\section{ANALYTICAL MODEL DEVELOPMENT}

The inner conductor of a $106 \mathrm{MHz}$ QWR is mechanically coupled with a coaxial reinforcing tube by a damper assembly as shown in Fig. 1. The principle of the damper operation is described in Ref. [10]. Both the inner conductor and reinforcing tube are fixed at the root. The damper assembly rests on the frictional brass plate terminated bottom of the reinforcing tube. It consists of three parts: a brass damper weight, a stainless steel conical base, and three coupling pins. The damper weight provides the main contribution of normal load that applies to the frictional plate from the damper. The three pins symmetrically spaced $120^{\circ}$ on azimuth are free to slip over both the inside of the QWR inner conductor and the damper stainless steel base. They are in contact by gravity from the brass load, which enables motion transfer. Consequently, the vibration energy is dissipated by friction when the damper moves on the frictional plate.

In either orthogonal direction, the whole system can be presented as a damped double mass-spring oscillator (see Fig. 2) in a frictional contact. A single contact friction model
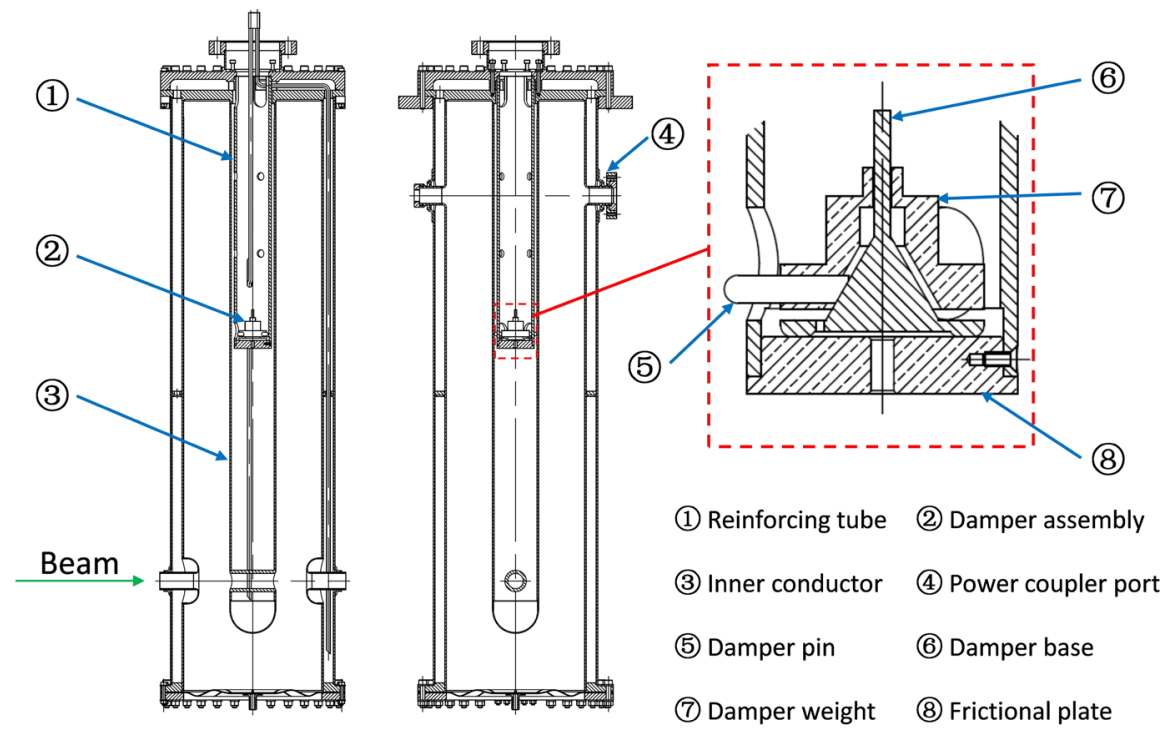

FIG. 1. The drawing of $106 \mathrm{MHz}$ QWR with details of the damper assembly. 


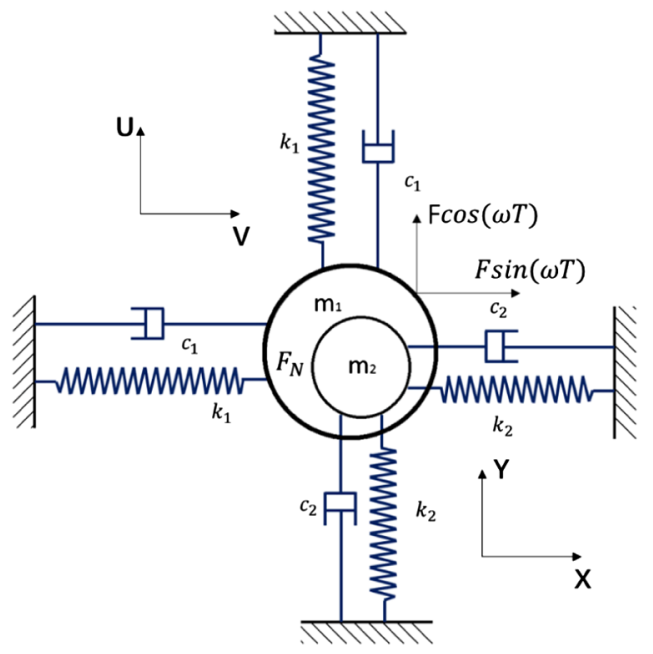

FIG. 2. Equivalent spring-mass model of a QWR installed with a damper.

is employed to capture the main characteristics of the system response. The tip displacement of the reinforcing tube has been scaled to that of the inner conductor by its fundamental mode vector modulus $\phi_{d}$ at the damper position. A harmonic drive simulates excitations from the ambient noise. A drive is acting in one direction, and the issue is said to be one dimensional with the motion back and forth, while more generally a two-dimensional motion is formed by multiple excitations acting in mutually orthogonal directions. The responses in both cases will be solved in sequence.

\section{A. One-dimensional motion}

The motion in the vertical direction with coordinate labels $U$ and $Y$ is considered only. For convenience, to solve a nonlinear problem with friction, all the lumped parameters in Fig. 2 are nondimensionalized with the following notations:

$$
\begin{aligned}
\epsilon & =\frac{m_{2}}{m_{1}}, \quad p=\frac{k_{2}}{k_{1}}, \quad q=\frac{F}{\phi_{d} \mu N}, \quad f_{N}=\frac{F_{N}}{\phi_{d} \mu N}, \\
\omega_{1}^{2} & =\frac{k_{1}}{m_{1}}, \quad \Omega=\frac{\omega}{\omega_{1}}, \quad u=\frac{k_{1} U}{\phi_{d} \mu N}, \quad y=\frac{k_{1} Y}{\phi_{d} \mu N}, \\
t & =\omega_{1} T, \quad \xi_{1}=\frac{c_{1}}{2 \sqrt{k_{1} m_{1}}}, \quad \xi_{2}=\frac{c_{2}}{2 \sqrt{k_{2} m_{2}}} .
\end{aligned}
$$

Here, $\mu$ is the sliding coefficient of friction, and $N$ is the normal force contributed by the entire damper assembly. For sliding friction, $f_{N}=1 . c_{1}$ and $c_{2}$ are viscous damping parameters defined by material properties of niobium and stainless steel, respectively.

The system is governed by two equations of motion:

$$
\begin{gathered}
u^{\prime \prime}+2 \xi_{1} u^{\prime}+u=q \cos (\Omega t)-f_{N}, \\
\epsilon y^{\prime \prime}+2 \xi_{2} \sqrt{\epsilon p} y^{\prime}+p y=f_{N},
\end{gathered}
$$

where the maximum response highly depends on the slip rate of the damper. When the forcing drive magnitude $q$ is relatively low, the damper purely sticks to the frictional plate underneath, and the entire system response is linear to the drive with a higher natural frequency, $\Omega_{1}=\sqrt{(1+p) /(1+\epsilon)}$. Equation (2) can be rewritten in a combined form:

$$
(1+\epsilon) u^{\prime \prime}+2\left(\xi_{1}+\xi_{2} \sqrt{\epsilon p}\right) u^{\prime}+(1+p) u=q \cos (\Omega t)
$$

The resonant response is given by

$$
A=\frac{q}{2\left(\xi_{1}+\xi_{2} \sqrt{\epsilon p}\right) \Omega_{1}} .
$$

The system is linear with an increasing drive until the maximum static friction is unable to maintain the coordinating motions:

$$
\sqrt{\left(p A_{1}-\epsilon A_{1} \Omega_{1}^{2}\right)^{2}+\left(2 \xi_{2} \sqrt{\epsilon p} \Omega_{1} A_{1}\right)^{2}}=1 .
$$

Plugging the result $A_{1}$ into Eq. (4) yields the drive magnitude $q_{1}$ at which the damper is activated.

When relative motion between the damper and the reinforcing tube is initiated, the response is no longer linearly varying with the drive. Assuming that the steadystate forced response is approximately harmonic, the displacement offset $u-y$ varies over a period of oscillation as illustrated in Fig. 3. Relative motion occurs on the paths with a nonzero slope. The maximum offset $\Delta$ is determined by the slip rate out of a period. In the case of the linear response, $\Delta=0$. The energy dissipated in the relative motion by friction per cycle is given by $4 \Delta$. Averaging in a cycle results in the energy balance in the steady state:

$$
4 \Delta=\pi\left(q-q_{1}\right) A_{1}
$$

The response amplitude is limited at $A_{1}$, since the presence of slip dissipates the portion of driven energy, accounting for the further exponential rise on $A_{1}$. This is in

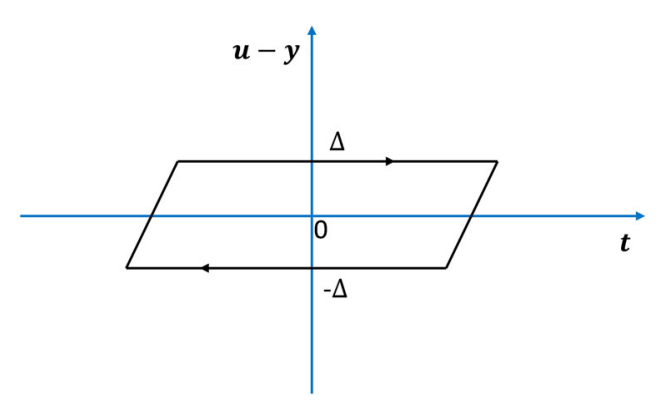

FIG. 3. Time-varying displacement offset $u-y$ over a period of oscillation. 
analogous to the presence of beam loading counteracting the exponential rise of a cavity field in the filling stage of an rf cavity. A flattop of the field is resulted over a macrobeam pulse. The partial slip in a cycle makes the damper work in an efficient way, which has been suggested in the study on aeroelastic instability control of engine airfoils $[16,17]$. This energy balance holds until the drive is sufficiently strong for the damper to be able to freely slip in all the cycle. Accordingly, $\Delta$ equals $A_{1}$ and yields

$$
q_{2}=\frac{4}{\pi}+q_{1}
$$

$q_{2}$ is an approximation to the critical condition and slightly higher than $4 / \pi$, since $q_{1} \ll 4 / \pi$. Beyond $q_{2}$, the system response is then dominated by the large-scale motion of the inner conductor, and the resonance is rapidly shifted towards its natural frequency. At resonance, $\Omega=1$, the energy balance relation similar to Eq. (6) is given by

$$
4 A+2 \pi \xi_{1} A^{2}=\pi q A .
$$

Here, the material damping of the reinforcing tube is reasonably ignored. The corresponding response is written in the form of

$$
A=\frac{q-\frac{4}{\pi}}{2 \xi_{1}}
$$

For a real solution, $q$ should be greater than $4 / \pi$. Let $A_{1}$ plug into the left part of Eq. (9), and a more conservative boundary to instability is obtained as

$$
q_{3}=\frac{4}{\pi}+2 \xi_{1} A_{1}
$$

From Eq. (4), $q_{3}$ is less than $q_{2}$. As the drive is sweeping over $4 / \pi$, the system response goes through a transition from constant $A_{1}$ to large-scale motion. Instead of using more complex analytical methods to the approach, a sharp corner $\left(q_{3}, A_{1}\right)$ represents the transition which originally in physics should be smooth. With this assumption and from Eqs. (4), (5), (7), and (9), the maximum response of the system at any harmonic excitation can be completely obtained.

\section{B. Two-dimensional motion}

Two harmonic drives in the quadrature phase are applied to $m_{1}$ as seen in Fig. 2. If the two drives are in phase, the problem is still in the scope of one dimension with the combined motion in back and forth. In quadrature phase thus is reasonably assumed. The two drives are first assumed at the same amplitude. The governing motion equation of system is written in a vector form:

$$
\begin{gathered}
\left(\begin{array}{l}
v \\
u
\end{array}\right)^{\prime \prime}+2 \xi_{1}\left(\begin{array}{l}
v \\
u
\end{array}\right)^{\prime}+\left(\begin{array}{l}
v \\
u
\end{array}\right)=q\left(\begin{array}{l}
\sin (\Omega t) \\
\cos (\Omega t)
\end{array}\right)-\boldsymbol{f}_{N}, \\
\epsilon\left(\begin{array}{l}
x \\
y
\end{array}\right)^{\prime \prime}+2 \xi_{2} \sqrt{\epsilon p}\left(\begin{array}{l}
x \\
y
\end{array}\right)^{\prime}+p\left(\begin{array}{l}
x \\
y
\end{array}\right)=\boldsymbol{f}_{N} .
\end{gathered}
$$

For $q \leq q_{1}$, the linear solution in Eq. (4) holds in the motion of either direction. A circular path could be visualized by the combined movements:

$$
\left(\begin{array}{l}
v \\
u
\end{array}\right)=\left(\begin{array}{l}
x \\
y
\end{array}\right)=A\left(\begin{array}{c}
\cos \left(\Omega_{1} t\right) \\
\sin \left(\Omega_{1} t\right)
\end{array}\right) .
$$

The friction force is in the form

$$
f_{N}=f_{N}\left(\begin{array}{c}
\cos \left(\Omega_{1} t+\delta\right) \\
\sin \left(\Omega_{1} t+\delta\right)
\end{array}\right)
$$

Here, the phase factor $\delta$ is determined by the relation $\tan (\delta)=2 \xi_{2} \sqrt{\epsilon p} \Omega_{1} /\left(\Omega_{1}^{2}-1\right)$.

For $q>q_{1}$, dynamic friction is present. A phase shift is required to allow relative motion:

$$
f_{N}=f_{N}\left(\begin{array}{c}
\cos \left(\Omega_{1} t+\delta+\phi\right) \\
\sin \left(\Omega_{1} t+\delta+\phi\right)
\end{array}\right)
$$

where its amplitude $\left|f_{N}\right|=1$. This implies a full slip all over a cycle as long as sliding occurs and certainly differs from one-dimensional motion in which a partial slip exists. With respect to the displacements of both masses, the integral in Eq. (14) over a period leads to the energy balance equation

$$
\pi A_{1}[\sin (\phi+\delta)-\sin (\delta)]=\pi A_{1}\left(q-q_{1}\right) .
$$

Here, in the integral, the displacements are given by

$$
\begin{aligned}
& \left(\begin{array}{l}
v \\
u
\end{array}\right)=A_{1}\left(\begin{array}{c}
\cos \left(\Omega_{1} t\right) \\
\sin \left(\Omega_{1} t\right)
\end{array}\right), \\
& \left(\begin{array}{l}
x \\
y
\end{array}\right)=A_{1}\left(\begin{array}{c}
\cos \left(\Omega_{1} t+\phi\right) \\
\sin \left(\Omega_{1} t+\phi\right)
\end{array}\right) .
\end{aligned}
$$

Equation (15) suggests that as in one-dimensional motion there is also a damping effective region though the damper slips fully in a cycle. The phase shift $\phi$ ranges in $(0, \pi / 2)$, within which a constant response at $A_{1}$ is kept and bounded at $\pi / 2$ :

$$
q_{2}=\cos (\delta)-\sin (\delta)+q_{1} .
$$

Typically, $\tan (\delta) \ll 1, \delta \approx 0$, and $q_{2}$ is approximated as

$$
q_{2} \approx 1+q_{1} \text {. }
$$

For $q>q_{2}$, large-scale motion at the resonance of $\Omega=1$ will dominate the response. Ignoring the negligible motion 
of the reinforcing tube results in a similar energy balance equation as Eq. (9):

$$
\pi A+2 \pi \xi_{1} A^{2}=\pi q A
$$

and yields the response

$$
A=\frac{q-1}{2 \xi_{1}} .
$$

$q>1$ is required for a valid solution. As the response reaches $A_{1}$, the corresponding drive $q_{3}$ is defined as a conservative boundary to instability:

$$
q_{3}=1+2 \xi_{1} A_{1} \text {. }
$$

Likewise, the transition connecting the instability region and the damping effective region is represented by a corner, $\left(q_{3}, A_{1}\right)$.

When the two drives are unequal at amplitude $(\underset{q-\Delta q}{q})$, an elliptical path will be formed. As for pure static friction coupling, corresponding to $q<q_{1}$, the solution is linear and Eq. (4) applies. In the range of $q_{1}<q<q_{1}+\Delta q$, the motion is linear in $U$, while in the orthogonal direction it follows the partial slip pattern. In terms of $q_{1}+\Delta q \leq q<$ $q_{3}+\Delta q$, the drives could be equivalent to a combination of a circular term and a linear one, $q\left(\begin{array}{c}\sin (\Omega t) \\ \cos (\Omega t)\end{array}\right)-\Delta q\left(\begin{array}{c}0 \\ \cos (\Omega t)\end{array}\right)$. The response will be the superposition of circular motion as given by Eq. (16) and linear motion with a displacement:

$$
\left(\begin{array}{c}
\Delta v \\
\Delta u
\end{array}\right)= \pm \Delta A\left(\begin{array}{c}
0 \\
\sin \left(\Omega_{1} t+\theta\right)
\end{array}\right)
$$

where $\tan (\theta)=\left(1-\Omega_{1}^{2}\right) / 2 \xi_{1} \Omega_{1}$, the sign is defined by that of $q-q_{3}$, and the amplitude

$$
\Delta A=\frac{\Delta q}{\sqrt{\left(1-\Omega_{1}^{2}\right)^{2}+\left(2 \xi_{1} \Omega_{1}\right)^{2}}}
$$

is typically far smaller than $A_{1}$. The path will be an ellipse slightly modified from a circle. Beyond $q_{3}+\Delta q$, large-scale motion in the inner conductor will dominate the system response [Eq. (20)], leading to instability eventually.

\section{Numerical results}

Numerical evaluations are performed for both onedimensional and two-dimensional models with the response curves indicated in Fig. 4. The linear response of the individual inner conductor, known as $\left(q / 2 \xi_{1}\right)$, is added to explicitly visualize the damping efficiency. The spring constants used in the evaluation were initially derived from a model of an idealized cantilever beam [18]. The extension effect at the root of the inner conductor due to finite rigidness, however, introduces an appreciable

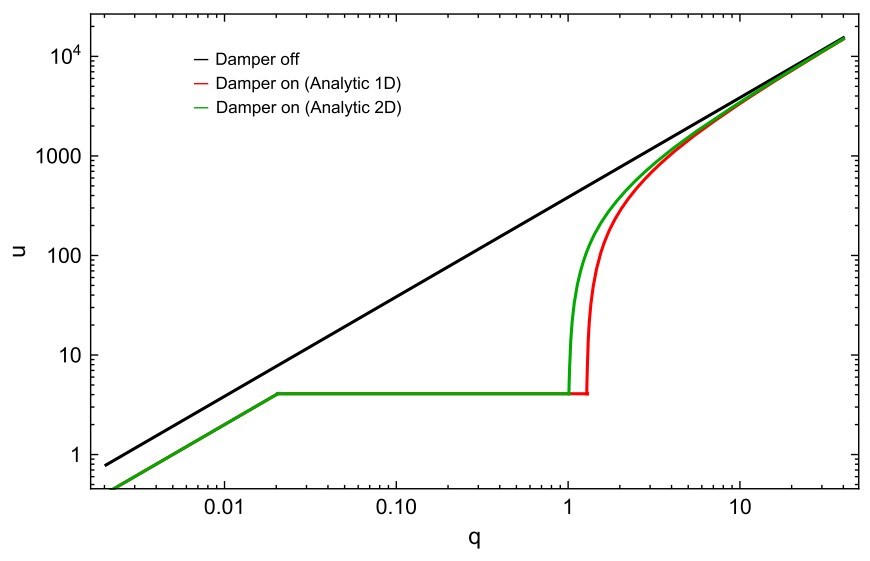

FIG. 4. Numerical evaluation of the analytic models for both $1 \mathrm{D}$ and 2D motion. The parameters used for evaluation: $k_{1}=1.11 \times 10^{5} \mathrm{~N} / \mathrm{m}, \quad m_{1}=0.61 \mathrm{~kg}, \quad \epsilon=0.073, \quad p=0.34$, $\phi_{d}=0.31, \quad \xi_{1}=0.0013$ (determined by measurements in Sec. IV), and $\xi_{2}=0.006$ [19].

difference to the correct eigenmode frequency of the fundamental mode (obtained either by a modal simulation or by measurement). Length adjustments of the cantilever beam for both the inner conductor and reinforcing tube were applied afterwards to take the extension effect into account. The physical displacement and driving force should be scaled from the numerical result by the factors of $\phi_{d} \mu N / k_{1}$ and $\phi_{d} \mu N$, respectively. Figure 4 indicates that an effective damping region exists in both response curves where none of the resonant mode is completely developed due to the presence of slip. Additional damping in the fully stick stage benefits from slightly higher resonance $\Omega_{1}$ and an enhanced natural damping ratio $\xi_{1}+\xi_{2} \sqrt{\epsilon p}$. As for the free slip stage, the damping efficiency degrades drastically with an increasing drive, ending with negligible friction damping resisting the large motion of the inner conductor. For less vibration in the damping effective region, one should either select a lighter damper or use a material combination with a smaller friction coefficient at the interface. Certainly, on the other hand, the system would have a lower stability boundary $4 \phi_{d} \mu N / \pi$ and a reduced range of effective damping as well.

\section{SIMULATIONS}

\section{A. Modal simulations}

Mechanical modal simulations for the full 3D model of $106 \mathrm{MHz}$ QWR with the damper either restrained or unrestrained were performed in ANSYS [20]. By means of finite element methods, the eigenmode frequency could be predicted precisely in the scope of linear response. Without the damper, the main mechanical vibration resonance of the inner conductor is $68.3 \mathrm{~Hz}$. It increases to $76.2 \mathrm{~Hz}$ as the overall stiffness is enhanced somewhat when the damper is fully stuck on the stainless steel reinforcing tube. The displacement patterns for both cases are shown in 

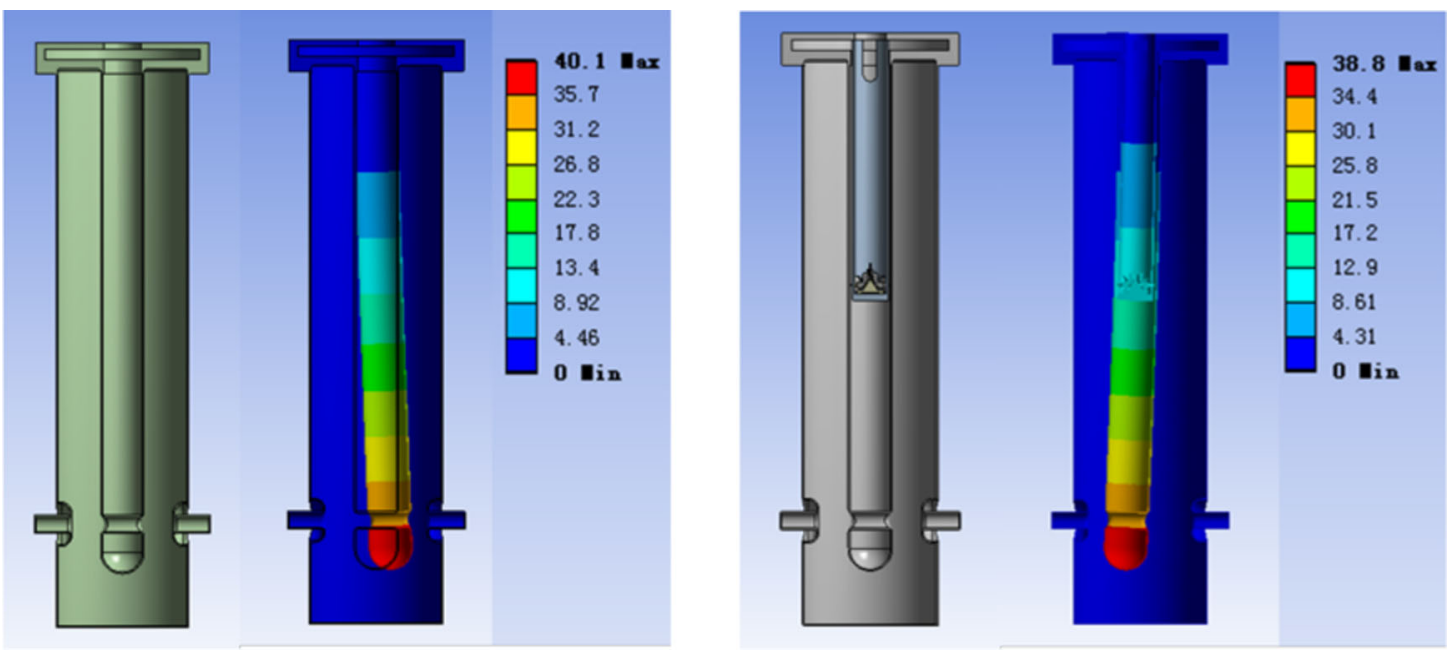

FIG. 5. Displacement pattern of the fundamental mode with scale in microns. Left: No damper; right: damper installed. The left pattern also includes the undeformed frame.

Fig. 5. Considering the operational condition at $4.2 \mathrm{~K}$, there will be about a 15\% rise on Young's modulus of niobium and a $7 \%$ rise for stainless steel $[21,22]$, with a corresponding $7.5 \%$ improvement on the modal frequency expected.

\section{B. Transient simulation in one dimension}

The numerical response of the QWR with a damper for external excitations can be obtained from a simulation using the ANSYS transient solver. The same cantilever beam model adopted in Sec. II is used to simplify fine geometric features in the full 3D model and avoids massive mesh generation. The two cantilever beams are connected by a contact element (CONTAT12) that represents the damper. This simplified model consists of 33 meshes. Multiple runs to identify the maximum response are enabled with an acceptable time cost in both frequency and drive sweeping of the harmonic drive, which is applied to the inner conductor tip. The sliding coefficient of dry friction 0.35 for a steel-brass interface is used [23]. The tip displacement of the inner conductor is recognized as the response and monitored over a wide range of drive. Figure 6 (left) illustrates the transient response at $q=$ 0.15 in the time domain. The flattop as a termination of the exponential rise is clearly seen. Figure 6 (right) shows both the simulation and analytical responses in good agreement. A slight rise of amplitude appears from the presence of slip in the simulation. The transition region as the drive crosses over the stability boundary, $4 / \pi$, is captured in the transient simulation. The evolutionary process of a slip rate with an increasing drive is well demonstrated in a series of plots of transient displacements $(u, y, u-y)$ in Fig. 7. The motion of the reinforcing tube is gradually out of tone with respect to that of the inner conductor and becomes negligible when the slip rate increases to the maximum. By rolling the time axes into one period, the displacement offset $u-y$ becomes the same as Fig. 3 .
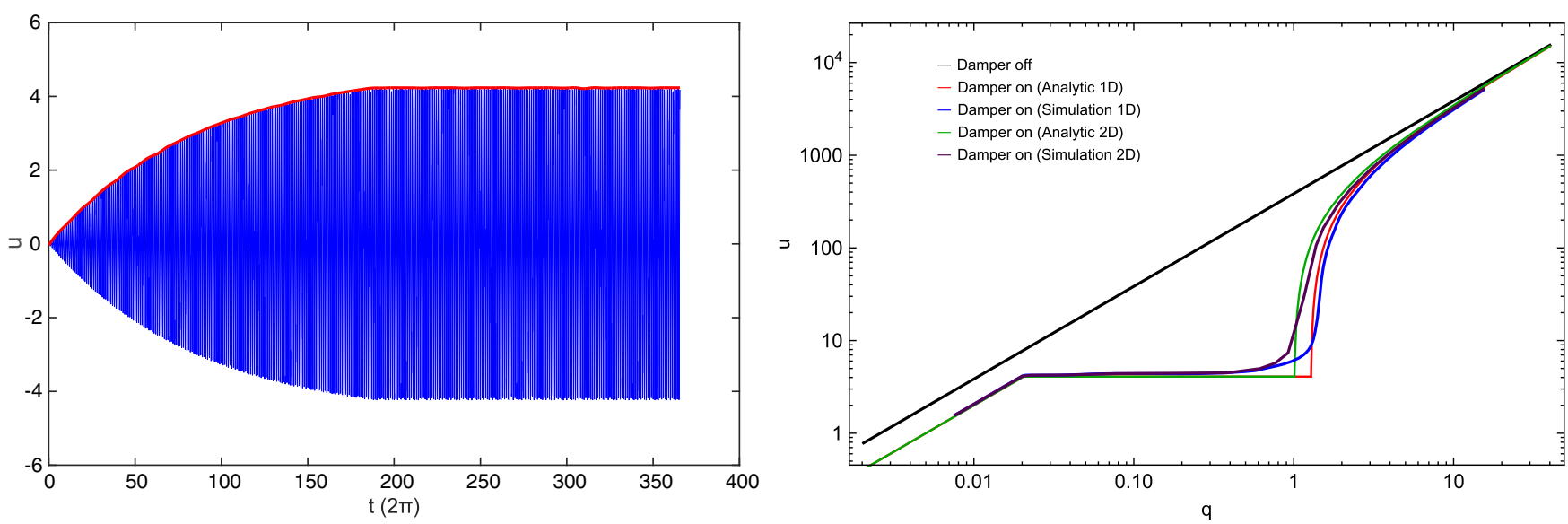

FIG. 6. Left: Transient response at $q=0.15$ varying with time- - the envelope is marked in red; right: simulation responses (1D and 2D) with the damper on-plots are added to Fig. 4 with a scale factor of $\phi_{d} \mu N / k_{1}$ and $\phi_{d} \mu N$. 

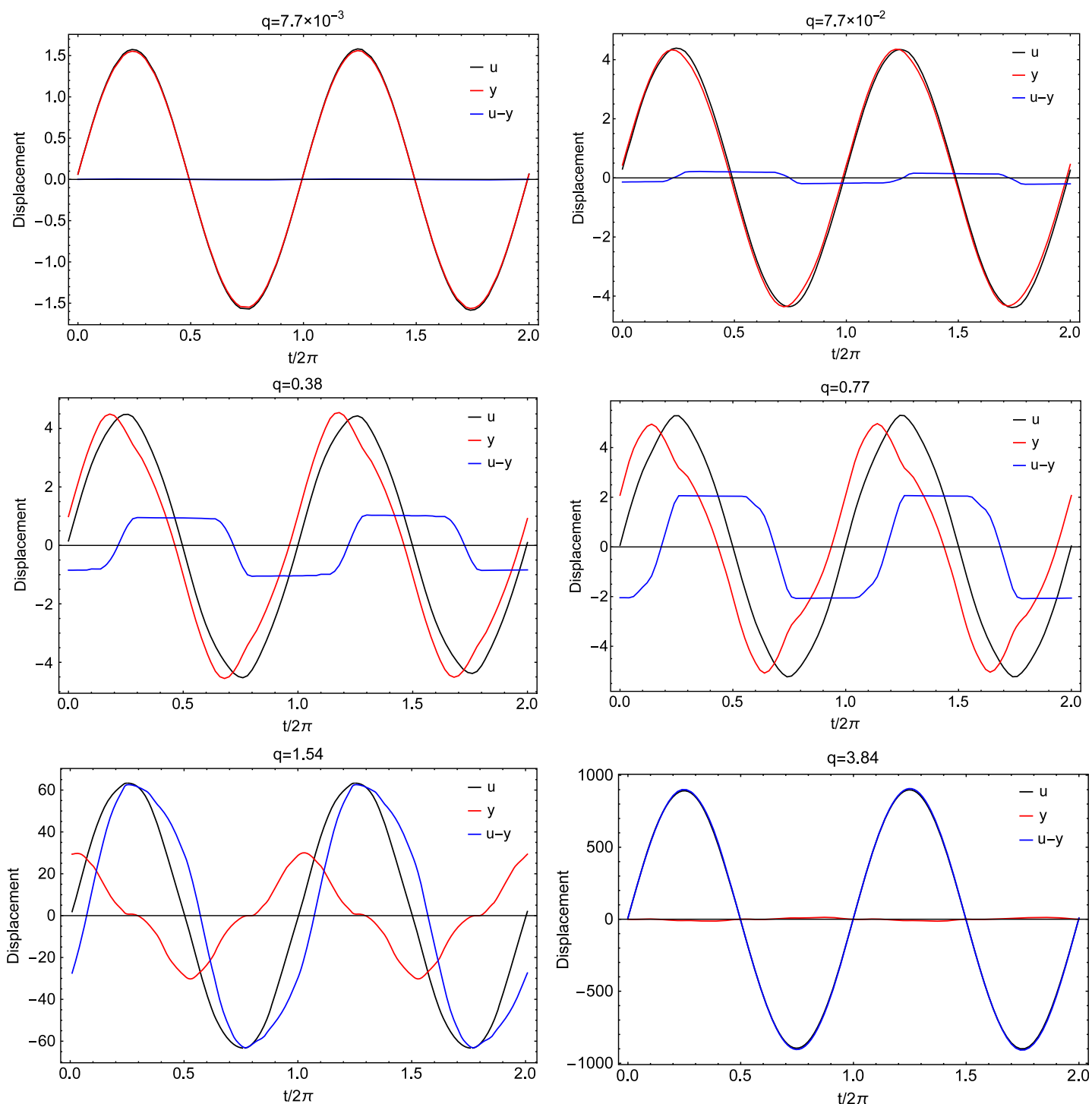

FIG. 7. Normalized displacements $(u, y, u-y)$ over time with an increasing drive $(q)$.
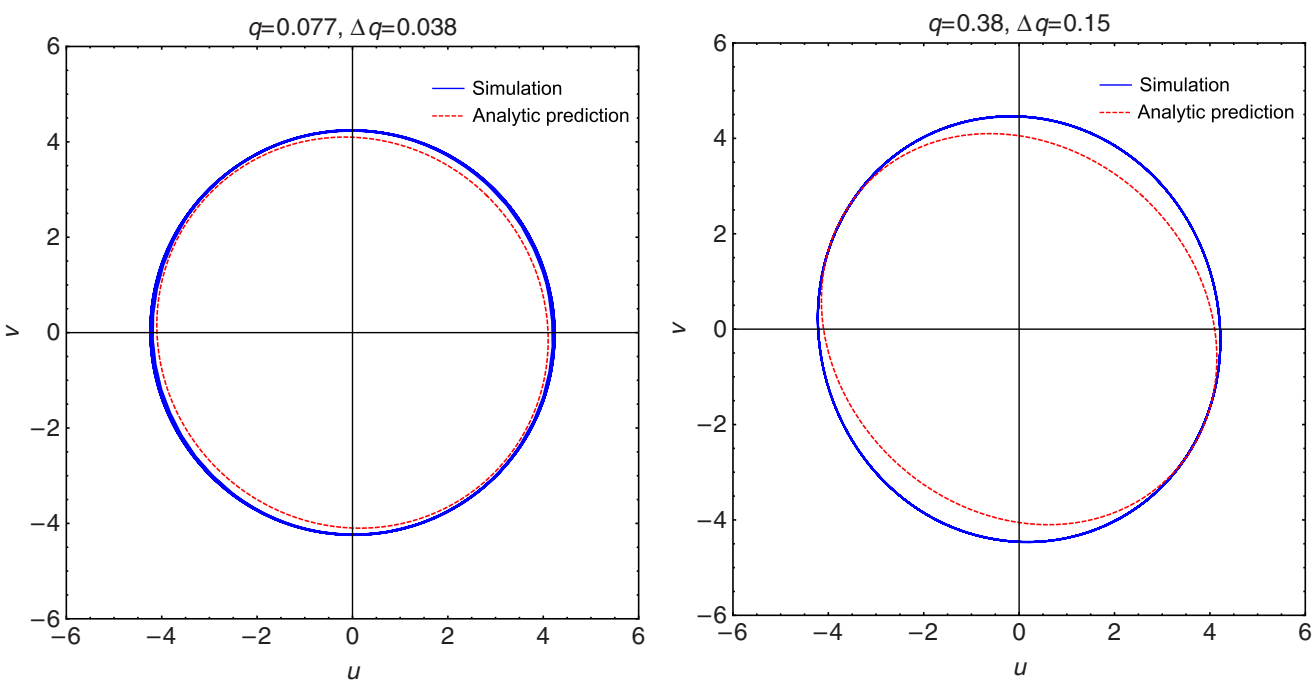

FIG. 8. Ellipse path formed under drives at unequal amplitude. 


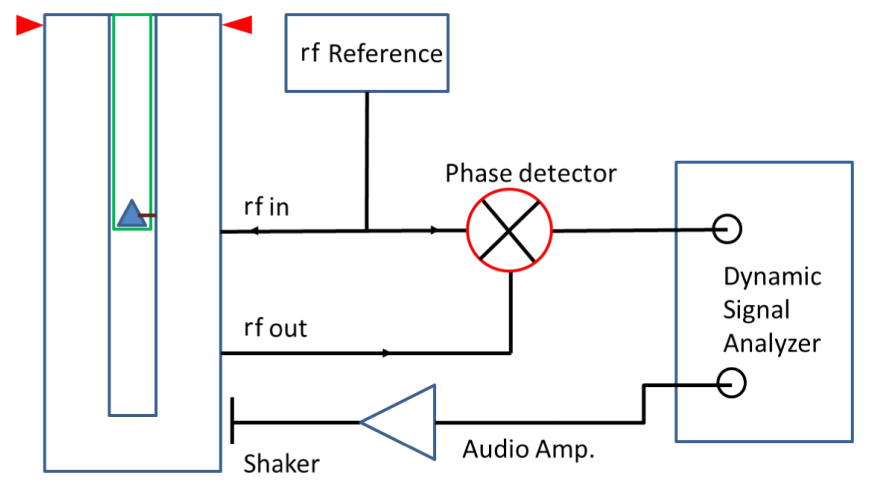

FIG. 9. Schematic of the test bench.

\section{Transient simulation in two dimensions}

The same procedure of simulation is performed to compute the maximum response in two-dimensional motion. The element of CONTAT12 is replaced with the more advanced one, CONTAT52, which is capable of solving the two-dimensional friction problem. In Fig. 6 (right) is shown the simulation response by sweeping a circular drive. The lower boundary of the damping effective range with an offset of 0.27 to the one-dimensional case is consistent with the analytical prediction $\frac{4}{\pi}-1$. Under an elliptical drive in the damping effective range, the simulated paths as shown in Fig. 8 compare well with the predictions by Eqs. (16) and (22). The response is mainly determined by the condition in Eq. (5) activating the damper, and thus the path is visually closer to a circle.

\section{EXPERIMENTAL VERIFICATION}

Harmonic response measurements on a spare $106 \mathrm{MHz}$ QWR were conducted to experimentally verify the predictions from both the analytical model and simulations. The test bench was configured in the similar way as in Ref. [10] and placed in a class 1000 clean room for ambient stability. This allows us access to switch on and off the damper during the measurement. The cavity was vertically suspended on a rigid frame with a fixed support at the top. A mechanical shaker driven by an audio amplifier was linked with the cavity outer wall via a copper band to create a forced vibration. Only one-dimensional excitation was applied. An rf signal generator fed the cavity through the power coupler port. As a reference, its frequency had been tuned to the cavity resonance in the static state. The electromagnetic field in the cavity was picked up via another port. The induced cavity detuning with respect to the reference is proportional to the response of the forced vibration. The phase error $\Delta \varphi$ between the pickup rf signal from the cavity and reference is related to the detuning level $\Delta f$ by the formula

$$
\tan (\Delta \varphi)=2 Q_{L} \frac{\Delta f}{f_{r}}
$$

where $Q_{L}$ is the loaded quality factor of the cavity and $f_{r}$ is the reference frequency. This phase error was detected by a phase detector and further processed into the spectrum in a dynamic signal analyzer (DSA). The DSA also provided a sinusoidal signal to drive the audio amplifier. The setup schematic is depicted in Fig. 9. The conversion coefficient to the cavity detuning level from the measured voltage at the DSA was calibrated.

By sweeping the drive signal frequency, the resonance measurement was first taken on the cavity with the damper off. The frequency sweeping was performed manually with one single frequency driving at each time to avoid any possible interference. The fundamental mode is identified at $68 \mathrm{~Hz}$ with a quality factor of 378 , corresponding to a natural damping ratio of 0.0013 . Further decay time measurements to confirm this material property gave consistent results.

By fixing the drive frequency at $68 \mathrm{~Hz}$, the linear response of the cavity with the damper off was measured as a baseline. The drive magnitude for the shaker was swept over a wide range of 3 orders. This allows the investigation of all the predicted stages for the following measurements with the damper engaged to the cavity. Out of all the damper parameters, only the dependence on damper weight was examined for the practical concern of variable control. The original damper assembly has a weight of $120 \mathrm{~g}$. Lighter ones of 85 and $60 \mathrm{~g}$ were prepared by modifications only on the weight part, as seen in Fig. 10, while all the shapes and materials of contact surfaces were kept the same. All three dampers were measured consequently

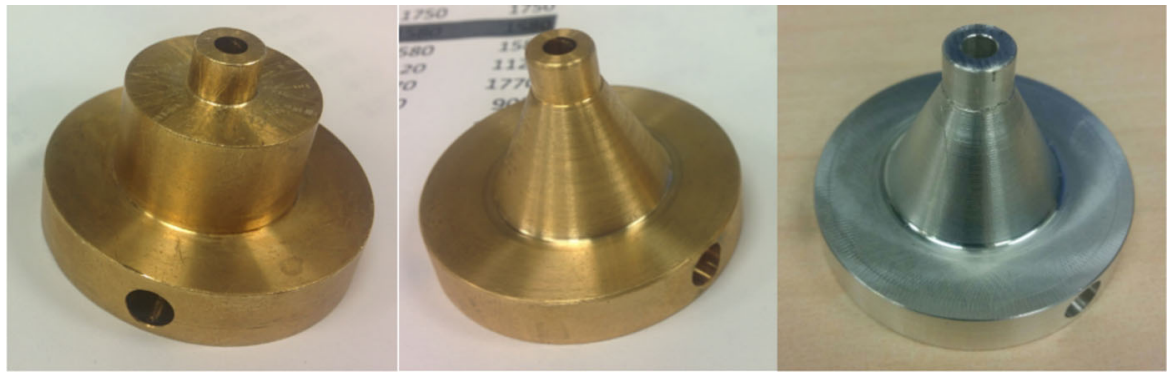

FIG. 10. From left to right, the weight part photos of the 120 (original), 85, and $60 \mathrm{~g}$ damper assembly. 


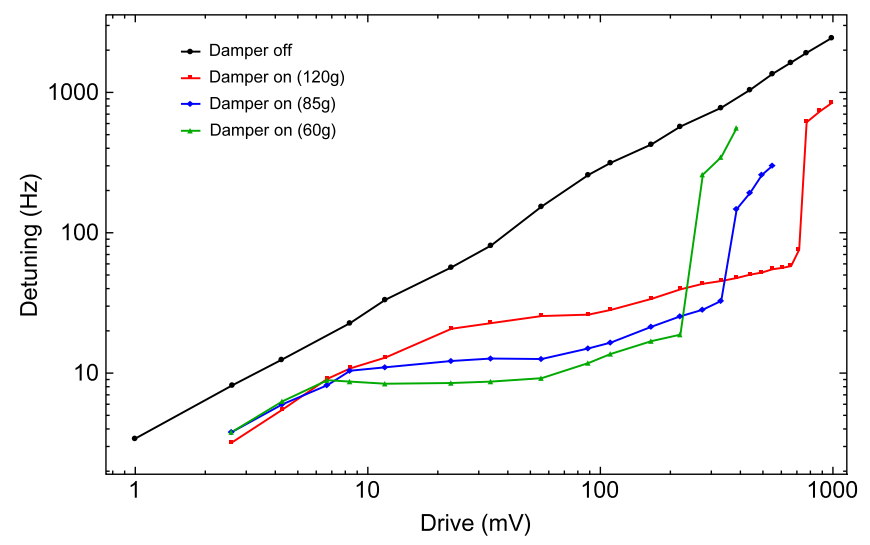

FIG. 11. Responses (represented by detuning) of drive sweeping with the damper off and with 120, 85, and $60 \mathrm{~g}$ dampers on.

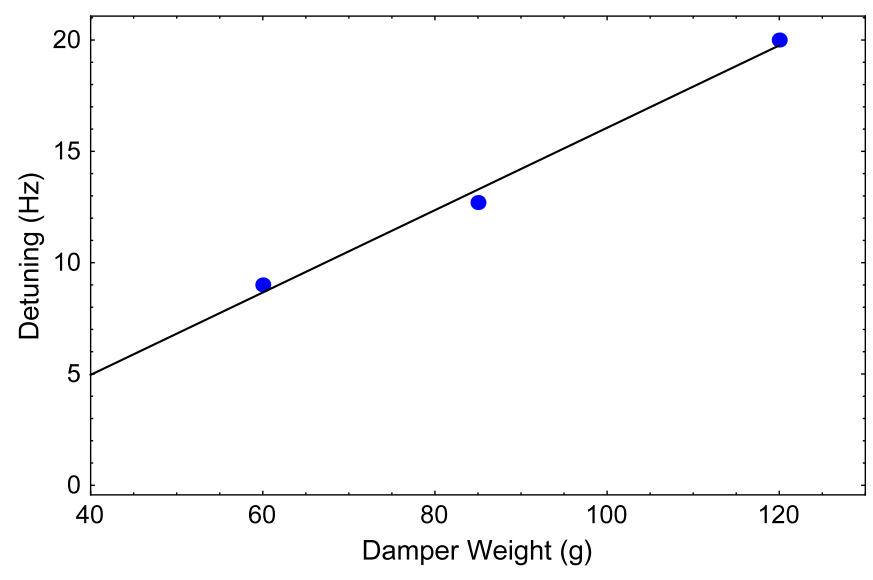

FIG. 12. Detuning level in the presence of slip for various damper weights.

without an interruption of force transmission between the shaker and cavity. The common mode for the coupled inner conductor and reinforcing tube as expected by the modal simulation was measured at $77 \mathrm{~Hz}$ with a sufficient lowlevel drive to avoid sliding. Figure 11 shows the response curves in comparison to that without the damper. The trend of the response curve with an increasing drive is consistent with the analytical prediction. In the partial slip stage, the amplitude rises, but a higher ramping rate than the simulation is seen for all three dampers. With the presence of slip, the lighter damper is more sensitive and provides better damping efficiency. In the damping effective range, reducing the damper weight leads to a proportional reduction in the maximum detuning level (Fig. 12).

\section{ONLINE MEASUREMENTS}

Online measurements of microphonics detuning were carried out on ISAC-II $106 \mathrm{MHz}$ QWRs. The phase error representing the cavity detuning level according to Eq. (24) was detected by the phase detector in the tuner loop of the
LLRF board for each cavity. It could be accessed via an additional output port of the phase error without interfering with the machine running. Time series data were recorded by the calibrated DSA with a maximum duration of $140 \mathrm{~s}$. The measured phase error is converted back to the detuning level, which is plotted as a probability distribution with Gaussian fitting (see Fig. 13 for an example). On average of seven cavities, the rms detuning level is $1 \mathrm{~Hz}$. Projected to the plot in Fig. 11, the corresponding excitation that leads to $1 \mathrm{~Hz}$ rms detuning is typically in the far left range, where the damper fully sticks to the reinforcing tube. The fundamental mode of inner conductor vibration is identified as $82 \mathrm{~Hz}$ from the phase error spectra (Fig. 14), as expected by the simulation. This frequency results from the common mode of the bundled inner conductor and reinforcing tube. The slight frequency rise with respect to that at room temperature is accounted for by the increased stiffness at $4.2 \mathrm{~K}$.

It can be drawn from online measurements that the excitations acting on the ISAC-II QWRs are normally in a moderate range, where the dampers are not activated. Only when the cavity is driven to large-scale vibration does the damper turn into the slip regime. As a result, the vibration is no longer on the track of exponential rising. Therefore, the maximum detuning is limited by the vibration amplitude at which the damper starts to slip. It is evident that it would be better to have dampers that initiate slip at lower excitations, particularly for the $106 \mathrm{MHz}$ QWRs. Reducing the damper weight is a simple way to improve the sensitivity as demonstrated in the bench test. For the original damper $(120 \mathrm{~g})$, slip onsets at a detuning level of about $20 \mathrm{~Hz}$ near the operational bandwidth, leading to out of lock in the phase loop. The $60 \mathrm{~g}$ damper reduces the maximum detuning level to $9 \mathrm{~Hz}$, which enables less overcoupling. For stable operation, the loaded bandwidth is better to cover the maximum detuning with an appropriate margin. As for the $10 \%$ margin to the maximum detuning level, $20 \mathrm{~Hz}$ of loaded bandwidth corresponds to a forward power of

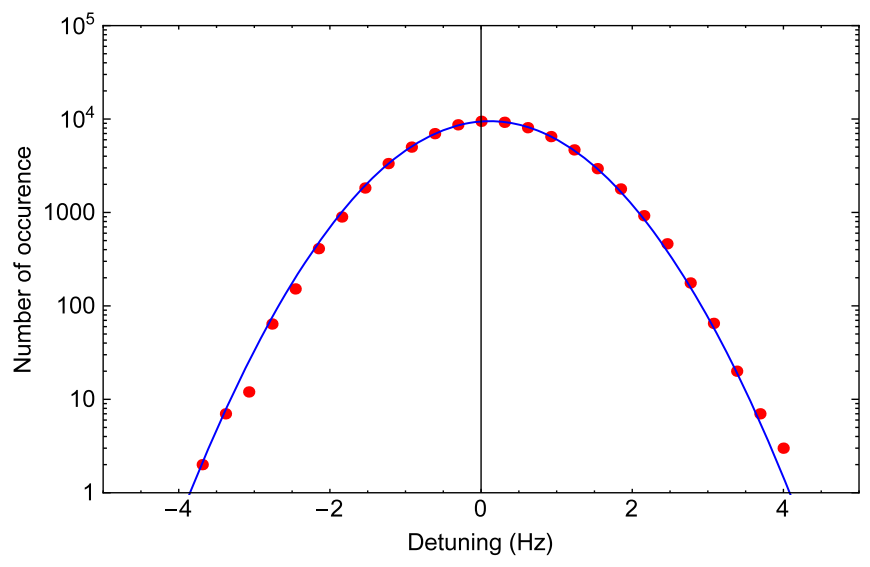

FIG. 13. An example of microphonics detuning probability. Gaussian fitting is included $\left(\sigma_{\mathrm{rms}}=0.9 \mathrm{~Hz}\right)$. 


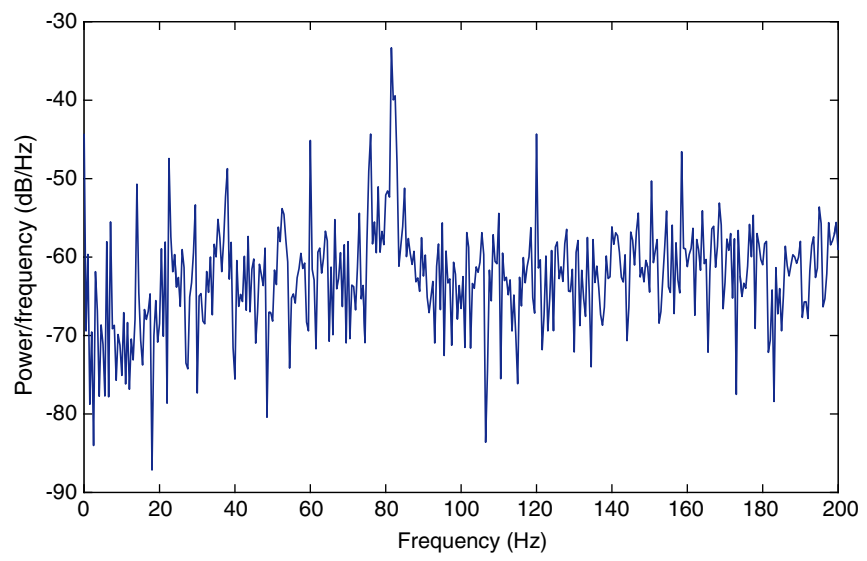

FIG. 14. Spectrum of the phase error record.

$100 \mathrm{~W}$. With the friction interface kept, the lightest weight that could be achieved by modifying the original design is $45 \mathrm{~g}$. The reduction of the maximum detuning could be speculated to be $63 \%$. At a margin of $30 \%, 20 \mathrm{~Hz}$ of loaded bandwidth takes $100 \mathrm{~W}$ of rf power as well, but more stable operations will be expected. Here, the speculation on reducing the operational forward power has carried over the measured data of the maximum detuning level from the bench test into the online measurement. A constant sliding coefficient of friction is assumed as well. There are actually two contrary effects involved to account for the change of the sliding coefficient of friction from room temperature to $4.2 \mathrm{~K}$, including the hydrodynamics of lubrication and temperature dependence, but which are roughly balanced on the variation for the steel-brass friction joint [24].

\section{CONCLUSION}

The damping of microphonics detuning in superconducting QWRs with frictional dampers is analytically addressed. In the scope of one-dimensional motion, the solution for a nonlinear system with friction is approached. The measure is then extended to the scenario of two-dimensional motion driven by multiple excitations in mutually orthogonal directions. The numerical results of the analytical model applied to the $106 \mathrm{MHz}$ QWR of ISAC-II are consistent with that predicted by the transient simulations in ANSYS. It has been shown that effective damping is maintained over a wide range of excitation. By increasing the excitation, the response amplitude holds at a relative low level along with a slight ramp in the response curve. The predicted characteristics of the response have been successfully demonstrated in the bench tests.

The response variation in the slip regime is highlighted in this work. The detuning measurements in the operational condition indicate that the excitation imposed on the QWRs is normally below the threshold level to start slip. When large-scale vibration of the QWR inner conductor is developed from environment noise, the presence of slip curtails amplitude growth. The sensitivity to trigger a slip could be adjusted by modifying the normal load of the damper. A lighter damper is suggested to limit the maximum detuning to a lower level as verified in the bench tests with various damper weights. The maximum detuning of $20 \mathrm{~Hz}$ for the original damper is reduced to $9 \mathrm{~Hz}$ for the lightest damper with half the weight. One-third of the weight could be achieved by modifying the original design with a speculated reduction of the maximum detuning level by $63 \%$. Such an improvement would enable a reduction in the bandwidth with more than a $50 \%$ reduction of rf power. Further verification on the performance of a modified damper implemented in the running cavities will be done in future tests. The study in this paper for selecting damper parameters could be extended to those superconducting QWRs that also employ frictional dampers in other accelerators.

\section{ACKNOWLEDGMENTS}

Inspired discussion with Zhongyuan Yao, and assistance of Norman Muller and Bill Rawnsley are gratefully acknowledged. Work was supported by the National Research Council Canada and the Natural Sciences and Engineering Research Council of Canada.

[1] I. Ben-Zvi and J. M. Brennan, The quarter wave resonator as a superconducting linac element, Nucl. Instrum. Methods Phys. Res. 212, 73 (1983).

[2] A. Facco and J. S. Sokolowski, The bulk niobium resonators program at LNL, Nucl. Instrum. Methods Phys. Res., Sect. A 328, 275 (1993).

[3] R. E. Laxdal, B. Boussier, K. Fong, M. Laverty, A. K. Mitra, and V. Zviagintsev, ISAC-II QWR cavity characterizations and investigations, Physica (Amsterdam) 441C, 193 (2006).

[4] M. P. Kelly et al., Microphonics in the ATLAS upgrade cryomodule, in Proceedings of the fourteenth International Conference on RF Superconductivity, SRF'09, Berlin, Germany, 2009 (JACoW, Geneva, 2009), pp. 269-272.

[5] W. Hartung et al., Superconducting quarter-wave resonator cavity and cryomodule development for a heavy ion Re-accelerator, in Proceedings of the XXIV Linear Accelerator Conference, LINAC'08, Victoria, BC, Canada, 2008 (JACoW, Geneva, 2008), pp. 854-856.

[6] S. Verdu-Andres et al., Design and vertical tests of doublequarter wave cavity prototypes for the high-luminosity LHC crab cavity system, Phys. Rev. Accel. Beams 21, 082002 (2018).

[7] T. Xin et al., Design of a high-bunch-charge 112-MHz superconducting RF photoemission electron source, Rev. Sci. Instrum. 87, 093303 (2016).

[8] A. Facco and V. Zvyagintsev, Beam steering in superconducting quarter-wave resonators: An analytical approach, Phys. Rev. ST Accel. Beams 14, 070101 (2011).

[9] P. N. Ostroumov and K. W. Shepard, Correction of beamsteering effects in low-velocity superconducting quarterwave cavities, Phys. Rev. ST Accel. Beams 4, 110101 (2001). 
[10] A. Facco, Mechanical mode damping in superconducting low $\beta$ resonators, in Proceedings of the Eighth Workshop on RF Superconductivity, SRF'97, Abano Terme (Padova), Italy, 1997 (JACoW, Geneva, 1997), pp. 685-694.

[11] J. R. Delayen, Phase and amplitude stabilization of superconducting resonators, Ph.D. thesis, Engineering and Applied Science Division, California Institute of Technology, 1978.

[12] J. M. Bogaty et al., An improved phase-control system for superconducting low-velocity accelerating structures, in Proceedings of the 1989 Particle Accelerator Conference, Chicago, IL (IEEE, New York, 1989), pp. 1978-1980.

[13] V. Zvyagintsev et al., A room temperature, low power fast tuner for superconducting resonators, in Proceedings of the Eighth Workshop on RF Superconductivity, SRF'97, Abano Terme (Padova), Italy, 1997 (JACoW, Geneva, 1997), pp. 695-700.

[14] S. Simrock et al., First demonstration of microphonic control of a superconducting cavity with a fast piezoelectric tuner, in Proceedings of the 2003 Particle Accelerator Conference, Portland, OR (IEEE, New York, 2003), pp. 470-472.

[15] S. Ghosh, Method to reduce microphonics in superconducting resonators, Phys. Rev. ST Accel. Beams 10, 042002 (2007).
[16] J.H. Griffin, Friction damping of resonant stresses in gas turbine engine airfoils, J. Eng. Power 102, 329 (1980).

[17] A. Sinha and J. H. Griffin, Friction damping of flutter in gas turbine engine airfoils, J. Aircr. 20, 372 (1983).

[18] J. Kokavecz and A. Mechler, Spring constant of microcantilevers in fundamental and higher eigenmodes, Phys. Rev. B 78, 172101 (2008).

[19] J. Zhang, R. J. Perez, and E. J. Lavernia, Documentation of damping capacity of metallic, ceramic and metal-matrix composite materials, J. Mater. Sci. 28, 2395 (1993).

[20] ANSYS, http://ansys.com.

[21] P. E. Armstrong et al., Temperature dependence of the elastic stiffness coefficients of niobium (columbium), Trans. Am. Soc. Metals 236, 1404 (1966).

[22] C. Antoine et al., Physical properties of niobium and specifications for fabrication of superconducting cavities, Report No. FERMILAB-TM-2503-TD-06-048, FERMILAB, Batavia, IL, 2006.

[23] Tribology, http://www.tribonet.org.

[24] C. G. Dunckle, M. Aggleton, J. Glassman, and P. Taborek, Friction of molybdenum disulfide-titanium films under cryogenic vacuum conditions, Tribol. Int. 44, 1819 (2011). 